\title{
DIVERGENT TYPE QUASILINEAR DIRICHLET PROBLEM WITH SINGULARITIES
}

\author{
S. PILIPOVIĆ, D. SCARPALEZOS
}

\begin{abstract}
A quasilinear equation of divergent type with singular data and singular coefficients is approximated by a net of equations of the same type with enough regular coefficients and data. Solutions of the net of equations are obtained by the classical methods. Known a priory estimates are improved so that a net of solutions can be considered as a solution in an appropriate algebra of generalized functions.
\end{abstract}

\section{INTRODUCTION}

Many linear and non-linear problems with irregular data or irregular coefficients, have been analyzed by the mean of appropriate approximations through appropriate nets of $C^{\infty}$ functions. One possible approach leads to the framework of generalized function algebra, cf. [1], [2], [8], [18]. Especially, we point out the recent work in this direction, [5], [6], [10], [11], [12], in the analysis of linear and nonlinear PDE with rough coefficients and initial data. They considered such problems in algebras of generalized functions where approximated nets of solutions have the meaning as elements of algebras of generalized functions enough large to contain embedded Schwartz distributions.

Our paper follows such an approach. Actually, we continue our investigations of linear elliptic equations with singular coefficients and data in [15]. In this paper, we solve an irregular Dirichlet quasi-linear elliptic problem using Leray-Schauder fixed point theorem (cf. [14]) in the framework of nets of equations with a priori estimates controlled by the growth order of constants appearing in these estimates. We transfer and improve some results of the classical theory for a Dirichlet quasi-linear elliptic problem given in [7] in the frame of certain generalized function algebra related to a strongly and uniformly elliptic quasi-linear Dirichlet problem with singular coefficients and singular boundary conditions. We refer to [3], [13], [17], [16], [20] for the classical results concerning quasi-linear Dirichlet problems.

More precisely, our approach is adapted to certain spaces of generalized functions because we follow the use of Leray-Schauder fixed point theorem in [7], Chapter 11. In order to apply the fixed point theorem we reconsider a priori bounds which are given in [7] in Theorems 8.22, 8.24, 8.27 and 8.29 as well as in Lemma 8.23. The novelty in the procedure of our proof (although we follow the known one of [7]) and the improvements of bounds in quoted 
assertions of [7], are our main contribution in solving an irregular Dirichlet quasi-linear elliptic problem.

In order to explain our approach the following simple example from [15] is useful.

Example 1. Consider $\Delta u=0, u_{\mid \partial \Omega}=\phi_{\mid \partial \Omega}$, where $\Omega=B_{1}=:\left\{\left(x_{1}, x_{2}\right)\right.$ : $\left.x_{1}^{2}+x_{2}^{2}<1\right\}$, with two boundary data given by $\phi$ :

Case I: $\quad \phi\left(x_{1}, x_{2}\right)=\delta\left(x_{1}\right) \delta\left(x_{2}-1\right),\left(x_{1}, x_{2}\right) \in \mathbb{R}^{2}$,

Case II: $\quad \phi\left(x_{1}, x_{2}\right)=\delta\left(\left(x_{1}^{2}+x_{2}^{2}\right)^{1 / 2}-1\right),\left(x_{1}, x_{2}\right) \in \mathbb{R}^{2}$.

Let $\theta \in C_{0}^{\infty}(\mathbb{R}) \int \theta=1$, supp $\theta \in[-1,1]$.

With approximations

Case I: $\delta\left(x_{1}\right) \delta\left(x_{2}-1\right)=\frac{1}{\varepsilon^{2}} \theta\left(\frac{x_{1}}{\varepsilon}\right) \theta\left(\frac{x_{2}-1}{\varepsilon}\right),\left(x_{1}, x_{2}\right) \in \mathbb{R}^{2}, \varepsilon<1$ (assuming $\theta(0)=0)$,

Case II: $\delta\left(\left(x_{1}^{2}+x_{2}^{2}\right)^{1 / 2}-1\right)=\frac{1}{\varepsilon} \theta\left(\frac{\left(x_{1}^{2}+x_{2}^{2}\right)^{1 / 2}-1}{\varepsilon}\right), \quad\left(x_{1}, x_{2}\right) \in \mathbb{R}^{2}, \varepsilon<1$ (assuming $\theta(0)=1$ ),

in the respective cases, we replace the given boundary data with the respective family of data:

Case I: $\quad \phi_{\varepsilon \mid \partial \Omega}(t)=\frac{1}{\varepsilon^{2}} \theta\left(\frac{t}{\varepsilon}\right) \theta\left(\frac{\sqrt{1-t^{2}}-1}{\varepsilon}\right),|t| \leq \varepsilon, \quad \phi_{\varepsilon}=0$ on the rest of $\partial \Omega$, Case II: $\quad \phi_{\varepsilon \mid \partial \Omega}=1 / \varepsilon, \varepsilon<1$.

The Poisson formula implies that in Case I, a net of solutions $\left(u_{\varepsilon}\right)_{\varepsilon}$ converges in the sense of distributions in $B_{1}$ to the harmonic function

$$
\left(x_{1}, x_{2}\right) \mapsto C_{\theta} \frac{|x|^{2}-1}{x_{1}^{2}+\left(x_{2}-1\right)^{2}},\left(x_{1}, x_{2}\right) \in B_{1},
$$

with the boundary equals zero everywhere except point $(0,1)$ where the boundary value is infinity. The net of solutions $\left(u_{\varepsilon}\right)_{\varepsilon}$ determines a generalized function $\left[\left(u_{\varepsilon}\right)_{\varepsilon}\right]$. In Case II solutions are harmonic functions $u_{\varepsilon}\left(x_{1}, x_{2}\right)=$ $1 / \varepsilon, \varepsilon<1$, in $B_{1}$ with boundary values $1 / \varepsilon, \varepsilon<1$. Note that $\left[(1 / \varepsilon)_{\varepsilon}\right]$ is a generalized constant, as it will be explained in the second section.

We will show in Section 3 that nets of boundary data given above, satisfy a generalized slope condition in a uniformly convex domain $\Omega$. This slope condition will be used in the main theorem, Theorem 4, for the proof of the existence of a generalized solution for a quasilinear problem of second order with a highly singular boundary data. More precisely, we prove the existence of a family of solutions in the frame of an appropriate algebra of generalized functions, in the so-called Colombeau type extensions of spaces $C^{k, \alpha}(\bar{\Omega})$ and $C^{\infty}(\bar{\Omega})$. Our approach is adapted to these spaces because we will follow the use of Leray-Schauder theorem as it is done in [7], Chapter 11. The nonlinear problem $a^{i, j}(D u) D_{i, j} u=0$ is transformed first to the net of non linear problems $a_{\varepsilon}^{i, j}\left(D u_{\varepsilon}\right) D_{i, j} u_{\varepsilon}=0, \varepsilon \in(0,1)$, with slowly increasing coefficients $a_{\varepsilon}^{i, j}(\varepsilon \rightarrow 0)$, then to a net of linear problems $a_{\varepsilon}^{i, j}(D v) D_{i, j} u_{\varepsilon}=0, \varepsilon \in(0,1)$ and, at the end, the problem is solved by the use of the Leray-Schauder fixed point theorem. The estimates of derivatives for a solution net $\left(u_{\varepsilon}\right)_{\varepsilon}$ are given by constants depending on $\varepsilon$. As it is mentioned, in order to apply 
the fixed point theorem we are led to revisit and improve a priori bounds which are given in [7] in Theorems 8.22, 8.24, 8.28 and 8.29 as well as in Lemma 8.23. In fact we give adequate versions of these theorems so that the bounds for norms $\left|u_{\varepsilon}\right|_{0, \alpha, \Omega}$ do not depend on $\alpha$.

Different growth rates of coefficients and initial data with respect to $\varepsilon \rightarrow 0$ lead to solutions in generalized ultradistribution type algebras ([19]) or in asymptotic type algebras $([4])$. Our choice of the growth rate is accommodated to the simplest exposition within Colombeau type algebras.

We note that a wide range of irregular problems can be modulated and discussed using the same method. In fact, in this paper we consider very simple form of a quasilinear Dirichlet problem in order to point out the difficulties which are brought into the equation through strong singularities of coefficients and initial data.

\section{Colombeau extension}

First, we recall the definition of the Colombeau type extention $\mathcal{G}(E)$, where $E$ is a vector space on $\mathbb{C}$ with an increasing sequence of seminorms $\mu_{n}, n \in \mathbb{N}$. The space of moderate nets $\mathcal{E}_{M}(E)$, respectively, of null nets $\mathcal{N}(E)$, is constituted by nets $\left(r_{\varepsilon}\right)_{\varepsilon \in(0,1]} \in E^{(0,1]}$ with the properties

$$
\begin{gathered}
\qquad(\forall n \in \mathbb{N})(\exists a \in \mathbb{R})\left(\mu_{n}\left(r_{\varepsilon}\right)=\mathcal{O}\left(\varepsilon^{a}\right)\right), \\
\text { respectively, }(\forall n \in \mathbb{N})(\forall b \in \mathbb{R})\left(\mu_{n}\left(r_{\varepsilon}\right)=\mathcal{O}\left(\varepsilon^{b}\right)\right) .
\end{gathered}
$$

( $\mathcal{O}$ is the Landau symbol.) The quotient space $\mathcal{G}(E)=\mathcal{E}_{M}(E) / \mathcal{N}(E)$ with elements $\left[\left(f_{\varepsilon}\right)_{\varepsilon}\right],\left[\left(g_{\varepsilon}\right)_{\varepsilon}\right], \ldots$, (equivalence classes are denoted by $\left.[\cdot]\right)$ is called the Colombeau extension of $E$. Putting $v_{n}\left(r_{\varepsilon}\right)=\sup \left\{a ; \mu_{n}\left(r_{\varepsilon}\right)=\mathcal{O}\left(\varepsilon^{a}\right)\right\}$ and $e_{n}\left(\left(r_{\varepsilon}\right)_{\varepsilon},\left(s_{\varepsilon}\right)_{\varepsilon}\right)=\exp \left(-v_{n}\left(r_{\varepsilon}-s_{\varepsilon}\right)\right), n \in \mathbb{N}$, we obtain $\left(e_{n}\right)_{n}$, a sequence of ultra-pseudometrics on $\mathcal{E}_{M}(E)$ defining the ultra-metric topology (sharp topology) on $\mathcal{G}(E)$.

If $E=\mathbb{C}$ (or $E=\mathbb{R}$ ) and the seminorms are equal to the absolute value, then the corresponding spaces are $\mathcal{E}_{0}$ and $\mathcal{N}_{0} ; \mathcal{E}_{0}$ is an algebra and $\mathcal{N}_{0}$ is an ideal. As a quotient, one obtains Colombeau algebra of generalized complex numbers $\overline{\mathbb{C}}=\mathcal{E}_{0} / \mathcal{N}_{0}$ (or $\overline{\mathbb{R}}$ ). If a set $\Omega$ is open in $\mathbb{R}^{n}$ and $E=C^{\infty}(\Omega)$ is endowed with the usual sequence of seminorms (this is Schwartz space $\mathcal{E}(\Omega)$ ), then the above definition gives Colombeau simplified algebra $\mathcal{G}(\Omega)=$ $\mathcal{E}_{M}(\Omega) / \mathcal{N}(\Omega)([2],[18])$. Its elements are called generalized functions and we keep this name for elements of any space or algebra constructed as extensions of some space of functions $E$.

Then the embedding of compactly supported Schwartz distributions (elements of $\mathcal{E}^{\prime}(\Omega)$ ) is made through the convolution with a net of mollifiers $h_{\varepsilon}=\varepsilon^{-n} h(\cdot / \varepsilon)$ constructed by a rapidly decreasing function $h \in \mathcal{S}\left(\mathbb{R}^{n}\right)$ with the properties $\int h(t) d t=1, \int t^{m} h(t) d t=0, m \in \mathbb{N}^{n},|m|>0$. The embedding is given by

$$
f \mapsto\left[\left(\left.f * h_{\varepsilon}\right|_{\Omega}\right)_{\varepsilon}\right]
$$


By the sheaf properties of $\mathcal{D}^{\prime}(\Omega)$ and $\mathcal{G}(\Omega)$, this embedding is extended to $\mathcal{D}^{\prime}(\Omega) \rightarrow \mathcal{G}(\Omega)$.

Let $\Omega$ be a bounded open set in $\mathbb{R}^{n}$ and $\alpha \in(0,1]$. Recall ([7], p. 94), a domain $\Omega$ and its boundary are of $C^{k, \alpha}-$ class $0<\alpha \leq 1$, if at each point $x_{0} \in \partial \Omega$ there is a ball $B=B\left(x_{0}\right)\left(x_{0}\right.$ is the center) and a bijection $\psi: B \rightarrow D$ such that $\psi(B \cap \Omega) \subset \mathbb{R}_{+}^{n}, \psi(B \cap \partial \Omega) \subset \partial \mathbb{R}_{+}^{n}$, and $\psi \in$ $C^{k, \alpha}(B), \psi^{-1} \in C^{k, \alpha}(D)$. A domain $\Omega$ has a boundary portion $T \in \partial \Omega$ of $C^{k, \alpha}$ - class if at each point $x_{0} \in T$ there is a ball $B\left(x_{0}\right)$ in which the above conditions are satisfied and $B\left(x_{0}\right) \cap \partial \Omega \subset T$.

We will consider Colombeau type extensions in cases $E=C^{k, \alpha}(\bar{\Omega}), k \in \mathbb{N}$ and $E=C^{\infty}(\bar{\Omega})$ and norms

$$
\begin{gathered}
|f|_{k, \Omega}=\sup \left\{\left|f^{(p)}(x)\right| ;|p| \leq k, x \in \Omega\right\}, \\
|f|_{k, \alpha, \Omega}=|f|_{k, \Omega}+[f]_{k, \alpha, \Omega}, k \in \mathbb{N}_{0},
\end{gathered}
$$

where, for $f \in C^{\infty}(\bar{\Omega}), k \in \mathbb{N}_{0}$,

$$
[f]_{k, \alpha, \Omega}=\sup \left\{\frac{\left|f^{(p)}(x)-f^{(p)}(y)\right|}{|x-y|^{\alpha}} ; x, y \in \Omega, x \neq y,|p|=k\right\} .
$$

The completion of $C^{\infty}(\bar{\Omega})$ with respect to the norm $|\cdot|_{k, \alpha, \Omega}$ defines $E_{k}=$ $C^{k, \alpha}(\bar{\Omega}), k \in \mathbb{N}$. Recall, if $k+\alpha>k^{\prime}+\alpha^{\prime}$, then the imbedding of $C^{k, \alpha}(\bar{\Omega})$ into $C^{k^{\prime}, \alpha^{\prime}}(\bar{\Omega})$ is a compact linear operator.

Note that the sequences of norms $|\cdot|_{k, \alpha, \Omega}, k \in \mathbb{N}$ and $|\cdot|_{k, \Omega}, k \in \mathbb{N}$ define the same uniform structure on $C^{\infty}(\bar{\Omega})$.

In case $E=C^{\infty}(\bar{\Omega})$, we need one more construction. Let $\left(g_{\varepsilon}\right)_{\varepsilon}$ be a net in $C^{0, \alpha}(\bar{\Omega})$ such that

$$
g_{\varepsilon} \in C^{k, \alpha}(\bar{\Omega}), \varepsilon<\varepsilon_{k}, k \in \mathbb{N},
$$

where $\left(\varepsilon_{k}\right)_{k} \in(0,1)^{\mathbb{N}}$ strictly decreases to zero $\left(\left(\varepsilon_{k}\right)_{k} \downarrow 0\right)$. Denote by $\mathcal{E} C^{\infty}(\Omega)$ the space of such nets.

Two such nets are in relation, $\left(g_{\varepsilon}\right)_{\varepsilon} \sim\left(r_{\varepsilon}\right)_{\varepsilon}$, if

$$
g_{\varepsilon}=r_{\varepsilon}, \varepsilon<\varepsilon_{0}, \text { for some } \varepsilon_{0} \in(0,1) .
$$

This is an equivalence relation and with the corresponding classes in $\mathcal{E} C^{\infty}(\Omega) / \sim$, we define by $(2.1)$ spaces $\mathcal{E}_{M}[E], \mathcal{N}[E]$. Thus, equivalent elements $\left(g_{\varepsilon}\right)_{\varepsilon},\left(r_{\varepsilon}\right)_{\varepsilon} \in \mathcal{E} C^{\infty}(\Omega)$ define the same element of $\mathcal{E}_{M}[E]$ iff one of them satisfies (2.1). We define the corresponding Colombeau type space $\mathcal{G}[E]=\mathcal{E}_{M}[E] / \mathcal{N}[E]$. If $E=C^{\infty}(\bar{\Omega})$, we will show in the appendix that there exists a canonical isomorphism of $\mathcal{G}[E]$ onto $\mathcal{G}(E)$. Clearly, $\mathcal{G}(E)=\mathcal{G}[E]$ in case $E=C^{k, \alpha}(\bar{\Omega})$ since for any representative $\left(r_{\varepsilon}\right)_{\varepsilon}$ the same class is determined by a representative $\left(g_{\varepsilon}\right)_{\varepsilon}$, where $g_{\varepsilon}=0, \varepsilon>0$ and $g_{\varepsilon}=r_{\varepsilon}, \varepsilon \leq$ $\varepsilon_{0}$, for some $\varepsilon_{0}<1$.

Let us note that the use of $\mathcal{G}\left[C^{\infty}(\bar{\Omega})\right]$, actually of $\mathcal{E}_{M}\left[C^{\infty}(\bar{\Omega})\right]$, enable us to use in the sequel the theory of Banach spaces on parts of nets for $\varepsilon<\varepsilon_{k}, k \in \mathbb{N}($ cf. $(2.2))$. 


\section{Dirichlet problem}

3.1. Assumptions and examples. We will consider a quasi-linear Dirichlet problem, a strictly and uniformly elliptic equation whose coefficients and data lack regularity assumptions. We will impose additional assumptions related to irregularities carried into equations and formulate the problem through nets of equations and corresponding assumptions. Our main reference is [7].

Let $\left(\mathcal{Q}_{\varepsilon}\right)_{\varepsilon}$ be a net of elliptic nonlinear operators of divergent type of the form

$$
\mathcal{Q}_{\varepsilon}(u)=\operatorname{div} \mathbf{A}_{\varepsilon}(D u)=a_{\varepsilon}^{i, j}(D u) D_{i, j} u, \varepsilon<1,
$$

where $a_{\varepsilon}^{i, j}(p)=D_{p_{i}} A_{\varepsilon}^{j}(p), p=\left(p_{1}, \ldots, p_{n}\right) \in \mathbb{R}^{n}$.

In the case $n=2$, the procedure which will be given below, can be applied to a net $\left(\mathcal{Q}_{\varepsilon}\right)_{\varepsilon}$ of elliptic nonlinear operators of the form

$$
\mathcal{Q}_{\varepsilon}(u)=a_{\varepsilon}^{i, j}(x, u, D u) D_{i, j} u, u \in C^{\infty}(\bar{\Omega}) .
$$

We assume that $a_{\varepsilon}^{i, j}, \varepsilon \in(0,1)$, are smooth functions on the respective domains. Let $\lambda_{\varepsilon}$ and $\Lambda_{\varepsilon}$ denote the minimal and maximal eigenvalues of the matrix $\left(a_{\varepsilon}^{i, j}\right)$. We assume

$$
\begin{gathered}
0<\lambda_{\varepsilon}(x, t, p)|\xi|^{2} \leq a_{\varepsilon}^{i, j}(x, t, p) \xi_{i} \xi_{j} \leq \Lambda_{\varepsilon}(x, t, p)|\xi|^{2}, \\
p \in \mathbb{R}^{n}, \xi \in \mathbb{R}^{n} \backslash\{0\}, x \in \Omega, t \in \mathbb{R}, \varepsilon<1 .
\end{gathered}
$$

In the sequel, we will denote by $c(\varepsilon), \varepsilon \in(0,1)$ a net of functions determining the growth rate of constants in the bounds which are to follow. We will assume that it is a constant function or a function tending to zero as $\varepsilon \rightarrow 0$. In fact we will assume in our main Theorem 4 that

$$
|\log c(\varepsilon)|=\mathcal{O}(\log |\log \varepsilon|), \varepsilon \rightarrow 0 .
$$

The reason will be seen later since we are aimed to give a generalized function solution determined by a net of solutions and for this the Harnack type estimates involve necessary assumptions on $c(\varepsilon)$.

Assume additionally:

$$
\begin{gathered}
\left(\forall d \in \mathbb{N}_{0}^{n}\right)(\exists l \in \mathbb{R})(\exists a \in \mathbb{R}) \\
\sup \left\{\frac{\left|\partial_{x}^{d} a_{\varepsilon}^{i, j}(x, t, p)\right|}{(1+|t|+|p|)^{a}} ; x \in \bar{\Omega}, t \in \mathbb{R}, p \in \mathbb{R}^{n}\right\}=\mathcal{O}\left(c^{l}(\varepsilon)\right) . \\
(\exists C>0)(\exists \mu>0)(\exists b \in \mathbb{R}) \\
\frac{c^{\mu}(\varepsilon)}{C}(1+|t|+|p|)^{b} \leq \lambda_{\varepsilon}(x, t, p) \leq \Lambda_{\varepsilon}(x, t, p) \leq \frac{C}{c^{\mu}(\varepsilon)}(1+|t|+|p|)^{b}, \\
p \in \mathbb{R}^{n}, x \in \bar{\Omega}, t \in \mathbb{R}, \varepsilon<1 .
\end{gathered}
$$

If $\left(\mathcal{Q}_{\varepsilon}\right)_{\varepsilon}$ is of the form (3.3), then we exclude variables $x$ and $t$ in the conditions given above.

Note that (3.8) implies the uniform ellipticity

$$
\Lambda_{\varepsilon} / \lambda_{\varepsilon} \leq C^{2} c^{-2 \mu}(\varepsilon), \varepsilon<1 .
$$


Example 2. (i) Strongly and uniformly elliptic equations with coefficients not depending on $\varepsilon$ but with singular boundary data are the main source of examples (cf. Example 1).

(ii) Consider in $\mathbb{R}^{3}$ an equation formally of the form

$$
\mathcal{Q}(D u)=\left(1+\sum_{i=1}^{3} f\left(D_{i} u\right)\right) \Delta u,
$$

where $f$ is a locally integrable function on $\mathbb{R}^{3}$. With

$$
\mathbf{A}=\left(x_{1}+F\left(u_{x_{1}}\right), x_{2}+F\left(u_{x_{2}}\right), x_{3}+F\left(u_{x_{3}}\right)\right) \text { and } F^{\prime}=f,
$$

we have $\mathcal{Q}(D u)=\operatorname{div} \mathbf{A}(D u)$, that is equation of the form (3.3).

Even in a case $f=\delta$ we can give a meaning to this equation through the corresponding family of equations:

$$
Q_{\varepsilon}(D u)=\left(\frac{\psi\left(u_{x_{1}} / c(\varepsilon)\right)+\psi\left(u_{x_{2}} / c(\varepsilon)\right)+\psi\left(u_{x_{3}} / c(\varepsilon)\right)}{c(\varepsilon)}+1\right) \Delta u, \varepsilon<1,
$$

where $\psi$ is a compactly supported smooth function whose integral equals 1. Then, for $p \in \mathbb{R}^{3}$,

$$
\lambda_{\varepsilon}(p)=1, \Lambda_{\varepsilon}(p)=1+\frac{1}{c(\varepsilon)}\left(\psi\left(p_{1} / c(\varepsilon)\right)+\psi\left(p_{2} / c(\varepsilon)\right)+\psi\left(p_{3} / c(\varepsilon)\right)\right), \varepsilon<1 .
$$

Family of operators (3.10) is of the form (3.3) for which all the assumptions (3.3)-(3.6) hold.

We will study a Dirichlet problem with a slope condition adapted to the setting of generalized functions.

Definition 1. Let $\left(\phi_{\varepsilon}\right)_{\varepsilon} \in \mathcal{E}_{M}[E]$, where $E=C^{\infty}(\bar{\Omega})$, or $E=C^{k, \alpha}(\bar{\Omega})$ for some $k \in \mathbb{N}$. Let $\Gamma_{\varepsilon}=\left\{\left(x, z_{\varepsilon}\right), x \in \partial \Omega, z_{\varepsilon}=\phi_{\varepsilon}(x)\right\}, \varepsilon<1$. The boundary data on $\partial \Omega$ satisfies a moderate slope condition if for any $P_{\varepsilon} \in \Gamma_{\varepsilon}, \varepsilon<1$ there exist hyperplanes $\pi_{\varepsilon, P_{\varepsilon}}^{+}$and $\pi_{\varepsilon, P_{\varepsilon}}^{-}$defined by $z_{\varepsilon}=\pi_{\varepsilon, P_{\varepsilon}}^{+}(x)$ and $z_{\varepsilon}=\pi_{\varepsilon, P_{\varepsilon}}^{-}(x)$ such that

$$
\pi_{\varepsilon, P_{\varepsilon}}^{-}(x) \leq \phi_{\varepsilon}(x) \leq \pi_{\varepsilon, P_{\varepsilon}}^{+}(x), x \in \partial \Omega, \varepsilon<1,
$$

and such that for some $K>0$ and some $m \in \mathbb{R}$,

$$
\sup \left\{\left|D \pi_{\varepsilon, P_{\varepsilon}}^{+}(x)\right|,\left|D \pi_{\varepsilon, P_{\varepsilon}}^{-}(x)\right| ; x \in \partial \Omega, P_{\varepsilon} \in \Gamma_{\varepsilon}\right\} \leq K \varepsilon^{m}, \varepsilon<1 .
$$

Example 3. 1. In the case when $\Omega=\left\{(x, y) ; x^{2}+y^{2}<1\right\}$ and $\theta(x, y)=$ $\delta\left(\left(x^{2}+y^{2}\right)^{1 / 2}-1\right)$ (case II in Example 1), the slope condition simply follows with the estimate $1 / \varepsilon$.

2. Let now, for equation (3.3), $\Omega$ be any uniformly convex domain in $\mathbb{R}^{2}$ such that $\partial \Omega \ni P=(0,1)$ and $\phi(x, y)=\delta(x) \delta(y-1)$ as in case I in Example 1 (with $\Omega=B_{1}$ ). In order to have the generalized slope condition, we take corresponding delta nets in $x$-plane and $y$-plane as case I in Exampe 1 and obtain that the slope condition holds with $K \varepsilon^{-4}, \varepsilon<1$. 
In fact, second example in Example 3, is a very special case of the following one.

Example 4. If $\Omega$ is uniformly convex, of $C^{2}$ class and if $\left(\phi_{\varepsilon}\right)_{\varepsilon} \in \mathcal{E}_{M}\left(C^{k, \alpha}(\bar{\Omega})\right)$, $k>2$, then $\left(\phi_{\varepsilon}\right)_{\varepsilon}$ satisfies the moderate slope condition with respect to $\Omega$.

Recall, $\Omega$ is uniformly convex, if there exists $C>0$ such that for every point $P_{0} \in \partial \Omega$ there exists a hyperplane $\pi_{P_{0}}$ such that

$$
d\left(x, \pi_{P_{0}}\right) \geq C\left\|x-x_{0}\right\|^{2}, x \in \partial \Omega, \text { where } P_{0}=x_{0} .
$$

By, [9], Corollary 4.3, $\phi \in C^{1,1}(\bar{\Omega})$ is the necessary and sufficient condition for $\phi$ to satisfy the bounded slope condition on such a domain. In particular, if $\phi \in C^{2}(\bar{\Omega})$, then the above assertion follows by evaluating a determinant depending on second derivatives of $\phi$ (cf. [9], p. 505). Note that in [9] are considered more general assumptions equivalent with the slope condition. In our case we use one of results for the illustration of the theory.

3.2. Estimates for nets of solutions. We will give several results needed for the proof of our main theorem, Theorem 4.

First, we need Lemma 1 as an extension of Lemma 8.23 in [7].

Lemma 1. Let $\alpha \in(0,1)$. Let $\omega$ be a non-decreasing function on $\left(0, R_{0}\right]$ such that

$$
\omega\left(\tau_{\delta} R\right) \leq \gamma_{\delta} \omega(R)+\sigma_{\delta}(R), \quad R \leq R_{0}
$$

where for every $\delta<1, \sigma_{\delta}$ is a non-decreasing function, $0<\gamma_{\delta}<1, \gamma_{\delta} \rightarrow$ 1 as $\delta \rightarrow 0,0<\tau_{\delta}<1, \tau_{\delta} \rightarrow 1$ as $\delta \rightarrow 0$ and $\lim _{\delta \rightarrow 0} \frac{\log n \gamma_{\delta}}{\log n \tau_{\delta}}=c>1$. Then there exists $\delta_{0}$ such that

$\omega(R) \leq \frac{1}{\gamma_{\delta}}\left(\frac{R}{R_{0}}\right)^{\alpha} \omega\left(R_{0}\right)+\frac{1}{1-\gamma_{\delta}} \sigma_{\delta}\left(\left(\frac{R}{R_{0}}\right)^{\frac{\log \gamma_{\delta}-\alpha \log \tau_{\delta}}{\log \gamma_{\delta}}} \cdot R_{0}\right), R \leq R_{0}, \delta<\delta_{0}$

Proof. Let $R_{1}<R_{0}$ and $\delta \in(0,1)$ be fixed. We have $\omega\left(\tau_{\delta} R\right) \leq \gamma_{\delta} \omega(R)+$ $\sigma_{\delta}\left(R_{1}\right), R \leq R_{1}$ By iteration, this inequality gives

$\omega\left(\tau_{\delta}^{m} R\right) \leq \gamma_{\delta}^{m} \omega\left(R_{1}\right)+\sigma_{\delta}\left(R_{1}\right) \sum_{i=0}^{m-1} \gamma_{\delta}^{i} \leq \gamma_{\delta}^{m} \omega\left(R_{0}\right)+\sigma_{\delta}\left(R_{1}\right) \frac{1}{1-\gamma_{\delta}}, \quad R \leq R_{1}$.

Let $m$ be chosen so that $\tau_{\delta}^{m} R_{1}<R \leq \tau_{\delta}^{m-1} R_{1}$. This implies

$$
\begin{aligned}
\omega(R) & \leq \omega\left(\tau_{\delta}^{m-1} R_{1}\right) \leq \gamma_{\delta}^{m-1} \omega\left(R_{0}\right)+\frac{\sigma_{\delta}\left(R_{1}\right)}{1-\gamma_{\delta}} \\
& \leq \frac{1}{\gamma_{\delta}}\left(\frac{R}{R_{1}}\right)^{\frac{\log \gamma_{\delta}}{\log \tau_{\delta}}} \omega\left(R_{0}\right)+\frac{\sigma_{\delta}\left(R_{1}\right)}{1-\gamma_{\delta}}
\end{aligned}
$$

Let $\delta_{0}>0$ be chosen so that $\frac{\log \gamma_{\delta}}{\log \tau_{\delta}}>1+h, \quad h>0, \quad \delta<\delta_{0}$. We have $0<\alpha \frac{\log \tau_{\delta}}{\log \gamma_{\delta}}<1, \delta<\delta_{0}$. Let $\mu_{\delta}=\frac{\log \gamma_{\delta}-\alpha \log \tau_{\delta}}{\log \gamma_{\delta}}, \delta<\delta_{0}$. Note $\left(1-\mu_{\delta}\right) \frac{\log \gamma_{\delta}}{\log \tau_{\delta}}=\alpha$. 
Put $R_{1}=R_{0}^{1-\mu_{\delta}} R^{\mu_{\delta}}, \delta<\delta_{0}$. It follows

$$
\omega(R) \leq \frac{1}{\gamma_{\delta}}\left(\frac{R}{R_{0}}\right)^{\left(1-\mu_{\delta}\right)\left(\frac{\log \gamma_{\delta}}{\log \tau_{\delta}}\right)} \omega\left(R_{0}\right)+\frac{\sigma_{\delta}\left(R_{0}^{1-\mu_{\delta}} R^{\mu_{\delta}}\right)}{1-\gamma_{\delta}}
$$

i.e.

$$
\omega(R) \leq \frac{1}{\gamma_{\delta}}\left(\frac{R}{R_{0}}\right)^{\alpha} \omega\left(R_{0}\right)+\frac{1}{1-\gamma_{\delta}} \sigma_{\delta}\left[\left(\frac{R}{R_{0}}\right)^{\mu_{\delta}} R_{0}\right], R<R_{0} .
$$

This completes the proof of lemma.

Remark. The crucial fact of the next Theorem 1 is that $\alpha \in(0,1 / 2)$ is fixed and does not depend on $\varepsilon$. The direct application of Lemma 8.23 of [7] would imply that in Theorem 1 instead of fixed $\alpha$ we would have a net $\left(\alpha_{\varepsilon}\right)_{\varepsilon}$ tending to zero with $\varepsilon \rightarrow 0$. Because of that we had to prove Lemma 1. Thus, the changes in the proof of Theorem 8.22 of [7] (based on Lemma 8.23) realized through the next Theorem 1 are interesting in itself (see also $[3],[17])$. This theorem is strictly involved in the proof of Theorem 4, and for its proof we will use the following generalized weak Harnack inequality which will be explained in the proof of Theorem 4:

$$
R^{-n}\left\|u_{\varepsilon}\right\|_{L^{1}\left(B_{\eta R}(y)\right)} \leq C \exp \left(c^{m}(\varepsilon)\right) \inf _{x \in B_{R}(y)} u_{\varepsilon}(x),
$$

where $\eta \in(1,2)$.

The linearization of (3.3) (and similarly of (3.4)) is done by putting a function $v$ instead of $u$ in coefficients $a^{i, j}(D u)$ Thus, equation (3.3), with $\tilde{a}_{\varepsilon}^{i, j}(x)=a_{\varepsilon}^{i, j}(D v(x))$, is of the form

$$
L_{\varepsilon} u_{\varepsilon}:=D_{i}\left(\tilde{a}_{\varepsilon}^{i, j}(x) D_{j} u_{\varepsilon}\right)+\left(-\sum_{j=1}^{n} \sum_{p=1}^{n} \frac{\partial}{\partial x_{p}} \tilde{a}_{\varepsilon}^{i, j}(x)\right) D_{i} u_{\varepsilon}=0 .
$$

In comparison to (8.1) and (8.3) in [7], we have $b=0, d=0, \mathbf{f}=0, g=0$.

Theorem 1. Let $\alpha \in(0,1 / 2)$ and $\Omega$ be a bounded open set. Let $\left(L_{\varepsilon}\right)$ be of the form (3.12), and satisfy conditions (3.7), ( 3.8). If $\left(u_{\varepsilon}\right)_{\varepsilon} \in\left(W^{1,2}(\Omega)\right)^{(0,1)}$ satisfies $L_{\varepsilon} u_{\varepsilon}=0$ in $\Omega, \varepsilon<1$, then for every $\varepsilon<1, u_{\varepsilon}$ is locally continuous in Hölder sense and there exist $M>0$, such that for every $B_{0}=B_{R_{0}}(y) \subset \Omega$ and every $R \leq R_{0}$,

$$
\operatorname{osc}_{B_{R}(y)} u_{\varepsilon} \leq M R^{\alpha} R_{0}^{-\alpha}{ }^{o s c_{B_{0}}} u_{\varepsilon} .
$$

Proof. Let $\varepsilon<1$. One has to repeat the proof of Theorem 8.22, [7] with $M_{\eta_{\varepsilon} R}$ and $m_{\eta_{\varepsilon} R}$ instead of $M_{4 R}$ and of $m_{4 R}$ defined as supremum and infimum of $u_{\varepsilon}$ on the ball $B_{\eta_{\varepsilon} R}(y)$. Recall, $\eta_{\varepsilon} \in(1,2)$. We will use the notation $M_{\varepsilon, R}$ and $m_{\varepsilon, R}$ for the supremum and the infimum of $u_{\varepsilon}$ on the ball $B_{R}(y)$.

Now, similarly as in Theorem 8.22. of [7], we use (3.11) for $M_{\eta_{\varepsilon}}-u_{\varepsilon}$ and $u_{\varepsilon}-m_{\eta_{\varepsilon}}$ in $B_{R / 2+\eta_{\varepsilon} R / 2}$ and obtain

$$
R^{-n} \int_{B_{R / 2+\eta_{\varepsilon} R / 2}}\left(M_{\eta_{\varepsilon} R}-u_{\varepsilon}\right) d x \leq C \exp \left(c^{m}(\varepsilon)\right)\left(M_{\eta_{\varepsilon} R}-M_{\varepsilon, R}\right),
$$


DIVERGENT TYPE QUASILINEAR DIRICHLET PROBLEM WITH SINGULARITIES 9

$$
R^{-n} \int_{B_{R / 2+\eta_{\varepsilon} R / 2}}\left(u_{\varepsilon}-m_{\eta_{\varepsilon} R}\right) d x \leq C \exp \left(c^{m}(\varepsilon)\right)\left(m_{\varepsilon, R}-m_{\eta_{\varepsilon} R}\right)
$$

By addition

$$
M_{\eta_{\varepsilon} R}-m_{\eta_{\varepsilon} R} \leq C \exp \left(c^{m}(\varepsilon)\right)\left(M_{\eta_{\varepsilon} R}-m_{\eta_{\varepsilon} R}+m_{\varepsilon, R}-M_{\varepsilon, R}\right)
$$

This leads to

$$
\omega_{\varepsilon}(R)=o s c_{B_{R}(y)} u_{\varepsilon}=M_{\varepsilon, R}-m_{\varepsilon, R} \leq\left(1-\frac{1}{C \exp \left(c^{m}(\varepsilon)\right.}\right) \omega_{\varepsilon}\left(\tau_{\varepsilon} R\right),
$$

where we put $\tau_{\varepsilon}=1 / \eta_{\varepsilon}$.

Using (3.13) and the Lemma 1, with $\sigma=0, \delta=\varepsilon, \gamma_{\varepsilon}=1-\frac{1}{C \exp \left(c^{m}(\varepsilon)\right)}, \tau_{\varepsilon}=$ $\sqrt{\gamma_{\varepsilon}}$, we have $\mu_{\varepsilon}=1-\frac{\alpha}{2}$, and

$$
\omega(R) \leq \frac{1}{\gamma_{\varepsilon}}\left(\frac{R}{R_{0}}\right)^{\alpha} \omega\left(R_{0}\right), R \leq R_{0} .
$$

(Note that $m$ is a negative constant.) This finishes the proof of Theorem 1

For the next two theorems the notions of exterior and uniform exterior cone condition are used. Recall that this the uniform exterior cone condition means that there exists a finite rigid circular cone $V$ such that at every $x \in \partial \Omega$ there exists $V_{x}$, congruent to $V$, such that $\bar{\Omega} \cap V_{x}=x$ (see [7], p.205).

The assumption $\partial \Omega$ is of $C^{2, \alpha}$ - class implies that $\partial \Omega$ satisfies the uniform exterior cone condition on the boundary.

The next step is the extension of Theorem 8.27 in [7], where we use Lemma 1 again.

Theorem 2. Let $\alpha \in(0,1 / 2)$ and $\Omega$ be a bounded open set. Let $\left(L_{\varepsilon}\right)$ be of the form (3.12), and satisfy conditions (3.7), (3.8). If $\left(u_{\varepsilon}\right)_{\varepsilon} \in\left(W^{1,2}(\Omega)\right)^{(0,1)}$ satisfies $L_{\varepsilon} u_{\varepsilon}=0, \varepsilon<1$, in $\Omega$, and $\Omega$ satisfies an exterior cone condition at a point $x_{0} \in \partial \Omega$, then, for every $0<R \leq R_{0}$ and $B_{0}=B_{R_{0}}\left(x_{0}\right)$,

$$
{ }_{o s c_{\Omega \cap B_{R}}} u_{\varepsilon} \leq \operatorname{Cexp}\left(c^{m}(\varepsilon)\right)\left(R^{\alpha} R_{0}^{-\alpha}+\sigma_{\varepsilon}\left(\sqrt{R R_{0}}\right)\right), \varepsilon<1,
$$

where $\sigma_{\varepsilon}(R)=$ osc $_{\partial \Omega} \cap B_{R} u_{\varepsilon}, \varepsilon<1$.

Proof. Similarly as in Theorem 1, we come to the inequality

$$
o s \Omega_{\Omega_{R}} u_{\varepsilon} \leq \gamma_{\varepsilon} O s c_{\Omega_{\eta_{\varepsilon}}} u_{\varepsilon}+o s c_{\partial\left(\Omega_{\eta_{\varepsilon} R}\right)} u_{\varepsilon}
$$

where $\Omega_{R}=\Omega \cap B_{R}\left(x_{0}\right), \partial\left(\Omega_{R}\right) \cap B_{R}\left(x_{0}\right)$. Now the proof follows by the use of Lemma 1.

Theorem 2 leads to a reformulated Theorem 8.29 of [7]:

Theorem 3. Assume that $\Omega$ satisfies a uniform exterior cone condition on a portion $T$ of the boundary. Let $\alpha_{0}<1, K_{\varepsilon}=\mathcal{O}\left(\exp \left(c^{m}(\varepsilon)\right)\right.$ and

$$
{ }_{o s c} c_{\partial \Omega \cap B_{R}\left(x_{0}\right)} u_{\varepsilon} \leq K_{\varepsilon} R^{\alpha_{0}}, x_{0} \in T, R>0,
$$

Then for $\alpha \in\left(0, \alpha_{0} / 2\right], u_{\varepsilon} \in C^{\alpha}(\Omega \cup T)$ and, for every $\Omega^{\prime} \subset \subset \Omega \cup T$, 


$$
\left\|u_{\varepsilon}\right\|_{C^{\alpha}\left(\Omega^{\prime}\right)} \leq C \exp \left(c^{m}(\varepsilon)\right) \sup _{\Omega}\left|u_{\varepsilon}\right|, \varepsilon<1,
$$

where $C$ depends on $d^{\prime}=\operatorname{dist}\left(\Omega^{\prime}, \partial \Omega-T\right)$

3.3. Main theorem. Let $k \in \mathbb{N}$. The assumptions in the next theorem are:

$$
\alpha \in(0,1 / 2), E=C^{k+2, \alpha}(\bar{\Omega}),
$$

$\Omega$ is open and bounded, $\partial \Omega$ is of $C^{k+2, \alpha}-$ class

$$
c^{m}(\varepsilon)=\mathcal{O}(|\log \varepsilon|), \text { for all } m \in \mathbb{N} .
$$

For example, one can take $c(\varepsilon)=\log |\log \varepsilon|, \varepsilon<1$.

Theorem 4. Assume that $\left(\mathcal{Q}_{\varepsilon}\right)_{\varepsilon}$ is a net of strongly and uniformly elliptic operators of the form (3.3) or (3.4) with $a_{\varepsilon}^{i, j} \in C^{k+1}\left(\bar{\Omega} \times \mathbb{R} \times \mathbb{R}^{n}\right)$ satisfying (3.7) and (3.8) with $d \leq k+1$. Moreover, assume that $\left(\phi_{\varepsilon}\right)_{\varepsilon} \in \mathcal{E}_{M}[E]$, where $\partial \Omega$ satisfies a moderate slope condition with $\left(\phi_{\varepsilon}\right)_{\varepsilon}$. Then, there exists $\left(u_{\varepsilon}\right)_{\varepsilon} \in \mathcal{E}_{M}[E]$ such that

$$
\mathcal{Q}_{\varepsilon}\left(u_{\varepsilon}\right)=0,\left.u_{\varepsilon}\right|_{\partial \Omega}=\phi_{\varepsilon}, \varepsilon<1
$$

Proof We follow ideas of Chapter 11 in [7] but for a parametric dependent family of equations. Actually, we change this procedure at an essential point (in order to obtain a priory bounds for the derivatives of order $k$ of a solution) although the steps of the proof are the same as the corresponding steps of the proof in [7].

Let $v \in C^{k+1, \alpha}(\bar{\Omega})$ be fixed. Consider a family of operators $\mathcal{Q}_{\varepsilon}^{v}$ defined by

$$
\mathcal{Q}_{\varepsilon}^{v}(u)=a_{\varepsilon}^{i j}(x, v, D v) D_{i j} u, \varepsilon<1 .
$$

For fixed $\varepsilon, \mathcal{Q}_{\varepsilon}^{v}$ is a strictly and uniformly elliptic linear operator. From (3.7), (3.8) and the fact that the $k$-th derivative of $a_{\varepsilon}^{i, j}(x, v, D v)$ is a polynomial expression of $\partial_{x}^{i} a_{\varepsilon}^{i, j}(x, v, D v), \partial_{x}^{i} v$ and $\partial_{x}^{i} D v, i \leq k$, it follows

$$
\left|a_{\varepsilon}^{i, j}(x, v, D v)\right|_{k, \alpha, \Omega} \leq C_{0} c^{\mu_{0}}(\varepsilon)\left(1+|v|_{k, \alpha, \Omega}+|D v|_{k, \alpha, \Omega}\right)^{b_{0}}, \varepsilon<1,
$$

for some $C_{0}>0, \mu_{0} \in \mathbb{R}$, and $b_{0} \in \mathbb{R}$.

By the classical theory $([7])$, we know that the linear Dirichlet problem

$$
\mathcal{Q}_{\varepsilon}^{v}(u)=0 \text { in } \Omega, u_{\partial \Omega}=\sigma \phi_{\varepsilon},
$$

for every fixed $\sigma \in[0,1]$ and $\varepsilon \in(0,1)$ admits a unique solution $u_{\varepsilon}^{v, \sigma}$ (cf. [7], Theorems 6.6 and 6.19$)$ in $C^{k+2, \alpha}(\bar{\Omega})$ satisfying the estimate

$$
\left|u_{\varepsilon}^{v, \sigma}\right|_{2, \alpha, \Omega} \leq C_{\varepsilon, \sigma}\left(\left|u_{\varepsilon}^{v, \sigma}\right|_{0, \alpha, \Omega}+\left|\phi_{\varepsilon}\right|_{2, \alpha, \Omega}\right),
$$

where $C_{\varepsilon, \sigma}>0$ depends on $\Omega, \lambda_{\varepsilon}(x, v, D v)$ and $\Lambda_{\varepsilon}(x, v, D v)$. Inspecting the proof of Theorem 6.6 in [7], we have found in [15] that

$$
C_{\varepsilon, \sigma} \leq C \frac{\Lambda_{\varepsilon}^{3}}{\lambda_{\varepsilon}^{3}}, \varepsilon<1
$$


This and (3.17) (with $k=0$ ) imply that there exist $C_{1}>0, m_{1} \in \mathbb{R}$, and $b_{1} \in \mathbb{R}$ such that

$$
C_{\varepsilon, \sigma} \leq C_{1} c^{m_{1}}(\varepsilon)\left(1+|v|_{1, \alpha, \Omega}\right)^{b_{1}}, \varepsilon<1 .
$$

This leads to

$$
\left|u_{\varepsilon}^{v, \sigma}\right|_{2, \alpha, \Omega} \leq C_{1} c^{m_{1}}(\varepsilon)\left(1+\left|u_{\varepsilon}^{v, \sigma}\right|_{0, \alpha, \Omega}+|v|_{1, \alpha, \Omega}+\left|\phi_{\varepsilon}\right|_{2, \alpha, \Omega}\right)^{b_{1}}, \varepsilon<1 .
$$

Now using (3.17) and the same arguments as above related to the $c(\varepsilon)-$ polynomial growth of constants appearing in (3.18), we conclude that there exists $C>0, m \in \mathbb{R}$, and $p \in \mathbb{R}$ such that

$$
\left|u_{\varepsilon}^{v, \sigma}\right|_{k+2, \alpha, \Omega} \leq C c^{m}(\varepsilon)\left(1+\left|u_{\varepsilon}^{v, \sigma}\right|_{0, \alpha, \Omega}+|v|_{k+1, \alpha, \Omega}+\left|\phi_{\varepsilon}\right|_{k+2, \alpha, \Omega}\right)^{p}, \varepsilon<1 .
$$

(See Exercise 6.2 in [7])

Fix $\varepsilon<1$ and denote by $T_{\varepsilon, \sigma}^{k}$ the mapping

$$
T_{\varepsilon, \sigma}^{k}(v)=u_{\varepsilon}^{v, \sigma}, v \in C^{k+1, \alpha}(\bar{\Omega}) .
$$

If for every $\varepsilon<1$ there exists a fixed point $v=u_{\varepsilon}^{\sigma}$, then it verifies

$$
\left|u_{\varepsilon}^{\sigma}\right|_{k+2, \alpha, \Omega} \leq C c^{m}(\varepsilon)\left(1+\left|u_{\varepsilon}^{\sigma}\right|_{0, \alpha, \Omega}+\left|u_{\varepsilon}^{\sigma}\right|_{k+1, \alpha, \Omega}+|\phi|_{k+2, \alpha, \Omega}\right)^{p}, \varepsilon<1 .
$$

Using this and (3.19), starting with $k=0$, with another $C>0, m \in \mathbb{R}$ and $p \in \mathbb{R}$, we have

$$
\left|u_{\varepsilon}^{\sigma}\right|_{k+2, \alpha, \Omega} \leq C c^{m}(\varepsilon)\left(1+\left|u_{\varepsilon}^{\sigma}\right|_{0, \alpha, \Omega}+\left|D u_{\varepsilon}^{\sigma}\right|_{0, \alpha, \Omega}+\left|\phi_{\varepsilon}\right|_{k+2, \alpha, \Omega}\right)^{p} .
$$

Now we will prove a priori bounds for $\left|u_{\varepsilon}^{\sigma}\right|_{1, \alpha, \Omega}$, of the form

$$
\left|u_{\varepsilon}^{\sigma}\right|_{1, \alpha, \Omega} \leq S \exp \left(c^{s}(\varepsilon)\right), \varepsilon<1,(\text { for some } S>0, s \in \mathbb{R}) .
$$

Because of this condition, we had assumed (3.6). This leads to the bound $S_{1} \varepsilon^{-s_{1}}$, with suitable positive constants $S_{1}$ and $s_{1}$.

In proving this we follow [7], p. 282, steps I, II, III and IV, where I is the estimate of $\sup _{\Omega}\left|u_{\varepsilon}\right|$ in terms of its values on the boundary, II is the estimate of $\sup _{\partial \Omega}\left|D u_{\varepsilon}\right|$ in terms of $\sup _{\Omega}\left|u_{\varepsilon}\right|$, III is the estimate of $\sup _{\Omega}\left|D u_{\varepsilon}\right|$ in terms of $\sup _{\partial \Omega}\left|u_{\varepsilon}\right|$ and $\sup _{\Omega}\left|u_{\varepsilon}\right|$, and IV is the estimate of $\sup _{\Omega}\left[D u_{\varepsilon}\right]_{\alpha, \Omega}$ in terms of $\sup _{\Omega}\left|D u_{\varepsilon}\right|$ and $\sup _{\Omega}\left|u_{\varepsilon}\right|$.

By the maximum principle we obtain bounds of $\left|u_{\varepsilon}^{v, \sigma}\right|_{0, \alpha, \Omega}$ by $\left|u_{\varepsilon}^{v, \sigma}\right|_{0, \alpha, \partial \Omega}$ ( [7], Theorems 10.3, 10.4 and 10.9); this is I.

The slope condition is introduced because of II and it gives the polynomial bounds in $\varepsilon$ for $\sup \left\{\left|D u_{\varepsilon}(x)\right| ; x \in \partial \Omega\right\}$. (cf. Chapters 14 and 15 in [7]).

The proof of III is the same as in [7], p. 284, considering the weak solutions to

$$
D_{i} \bar{a}_{\varepsilon}^{i, j}(x)\left(D_{j} w_{\varepsilon}\right)=0,
$$

where

$$
\bar{a}_{\varepsilon}^{i, j}(x)=a_{\varepsilon}^{i, j}\left(D u_{\varepsilon}(x)\right) \text { and } w_{\varepsilon}=D_{k} u_{\varepsilon}, k=1, \ldots n .
$$

As in [7], we use the weak maximum principle and obtain

$$
\sup _{\Omega}\left|u_{\varepsilon}\right| \leq \sup _{\partial \Omega}\left|D u_{\varepsilon}\right| \leq K \varepsilon^{m}, \varepsilon<1,
$$


where $K \varepsilon^{m}$ is from the boundary slope condition.

The proof of IV is essential. We will give it only in the case of (3.3).

Following the same line of the proof as in [7], we have reformulated Theorem 8.22 as Theorem 1.

Considering a family of linear equations (3.12) $L_{\varepsilon} u_{\varepsilon}=0$, we start from the reformulation of Theorem 8.18, [7] for the net of equations. In the proof of such theorem with $\beta \neq-1$, all the constants appearing in the estimates are of $c(\varepsilon)$ - polynomial growth but for $\beta=-1$ one obtains a $c(\varepsilon)$ - polynomial estimate for $\log \left(u_{\varepsilon}+k_{\varepsilon}\right.$ ) (in our case $k_{\varepsilon}=0$ ). This leads to the weak Harnack type inequality (8.47) and (8.63) in [7] (see also[16]). With the same arguments as in [7], it follows that the constants in (8.47) and (8.63) of [7] are of the form $\exp \left(\Lambda_{\varepsilon} / \lambda_{\varepsilon}+R \nu_{\varepsilon}\right)$, where $\nu_{\varepsilon}$ is also of $c(\varepsilon)-$ polynomial growth. Assumption (3.9) implies that constants are of the form $C \exp \left(c^{m}(\varepsilon)\right)$, for some $C>0$ and $m \in \mathbb{R}$ not depending on $\varepsilon$.

Now we use (8.47) of [7]:

$$
R^{-n}\left\|u_{\varepsilon}\right\|_{L^{1}\left(B_{2 R}(y)\right)} \leq C \exp \left(c^{m}(\varepsilon)\right) \inf _{x \in B_{R}(y)} u_{\varepsilon}(x), \varepsilon<1,
$$

where $u_{\varepsilon} \in W^{1,2}(\Omega)$ is a solution to (3.12) and non-negative in the ball $B_{4 R}(y) \subset \Omega$.

By (3.23), we have (3.11):

$$
R^{-n}\|u\|_{L^{1}\left(B_{\eta_{\delta}}(y)\right)} \leq C \exp \left(c^{m}(\varepsilon)\right) \inf _{x \in B_{R}(y)} u(x),
$$

where $1<\eta_{\delta}<2$. The use of this inequality in Theorem 1 implies that for every $\varepsilon<1, u_{\varepsilon}$ is locally continuous in Hölder sense and there exist $M>0, m \in \mathbb{R}$ such that for every $B_{0}=B_{R_{0}}(y) \subset \Omega$ and every $R \leq R_{0}$

$$
{ }_{o s c_{B_{R}}(y)} u_{\varepsilon} \leq M \exp \left(c^{m}(\varepsilon)\right) R^{\alpha} R_{0}^{-\alpha}{ }^{o s c_{B_{0}}} u_{\varepsilon} .
$$

Now with the same assumptions we have the conclusion of reformulated version of Theorem 8.24 [7] but always with $\alpha$ not depending on $\varepsilon$ :

For every $\Omega^{\prime} \subset \subset \Omega$ there exist $M>0, m \in \mathbb{R}$ such that

$$
\left\|u_{\varepsilon}\right\|_{C^{\alpha}\left(\Omega^{\prime}\right)} \leq M \exp \left(c^{m}(\varepsilon)\right)\left\|u_{\varepsilon}\right\|_{L^{2}(\Omega)}, \varepsilon<1 .
$$

The assumption in Theorem 4: $\alpha<1 / 2$ implies that $\alpha_{0}=2 \alpha<1$ This was important for Theorem 3. Thus, Theorem 3 (reformulated Theorem 8.29 , [7]) enable us to continue with the proof of IV. It follows as it is described in [7], pp. 284-285, considering the weak solutions to (3.22). (First, we have to make necessary change of coordinates to arrive to portions of $x_{n}=0$ instead of portions of $\partial \Omega$. Then we have to use the Theorem 3 for the bounds of $w$ for the derivatives $D_{y_{k}} w, k=1, \ldots, n-1$ and the Morrey's estimate for $D_{y_{n}} w$, Theorem 7.19 in [7]. This completes the proof of (3.21).

Now by (3.20) (with some new constants $C$ and $m$ ), we have

$$
\left|u_{\varepsilon}^{\sigma}\right|_{k+1, \alpha, \Omega}<C \exp \left(c^{m}(\varepsilon)\right)\left(\left|u_{\varepsilon}^{\sigma}\right|_{1, \alpha, \partial \Omega}+\left|\phi_{\varepsilon}\right|_{k+2, \alpha, \Omega}\right), \varepsilon<1,
$$


where on the left side we put $k+1, \alpha$-norm although it holds for $k+2, \alpha$ -norm.

For every $\varepsilon<1, T_{\varepsilon, \sigma}^{k}$ is a continuous mapping from $C^{k+1, \alpha}(\bar{\Omega})$ into $C^{k+2, \alpha}(\bar{\Omega})$ and thus, a compact mapping from $C^{k+1, \alpha}(\bar{\Omega})$ into $C^{k+1, \alpha}(\bar{\Omega})$.

By (3.24) we have that every solution $u_{\varepsilon}^{\sigma}$, if exists, being the fixed point of $T_{\varepsilon, \sigma}^{k}$, satisfies the bound

$$
\left|u_{\varepsilon}^{\sigma}\right|_{k+1, \alpha, \Omega} \leq C_{k} \exp c^{m_{k}}(\varepsilon), \varepsilon<1
$$

for suitable $C_{k}>0$ and $m_{k}>0$. Now the assumption (3.15) implies the estimate

$$
\left|u_{\varepsilon}^{\sigma}\right|_{k+1, \alpha, \Omega} \leq C \varepsilon^{m}, \varepsilon<1 .
$$

Proposition 1 (i) in the Appendix, implies that that there exists $\left(u_{\varepsilon}\right)_{\varepsilon}$, a fixed point for $\left(T_{\varepsilon}^{k}\right)_{\varepsilon}$ ( with $\sigma$ equals 1 ) which satisfies (3.26). The theorem is proved.

Remark. Let us underline that in [7], step IV is realized "with some $\beta \in(0,1)$ " instead of our fixed $\alpha \in(0,1)$. And this is another difference of our procedure in relation to [7] since we already come to inequality (3.20) in a different way.

We give the existence of a solution of the Dirichlet problem extending $E=C^{\infty}(\bar{\Omega})$ to generalized function algebra.

Theorem 5. Let $\left(\mathcal{Q}_{\varepsilon}\right)_{\varepsilon}$ be a net of strongly and unifotmly elliptic operators of the form (3.3) or (3.4) with $a_{\varepsilon}^{i, j} \in\left(C^{\infty}\right)^{(0,1)}(\bar{\Omega})$ satisfying (3.7), (3.8). Let $\partial \Omega$ be of $C^{\infty}$ - class and let $\partial \Omega$ and $\left(\phi_{\varepsilon}\right)_{\varepsilon} \in \mathcal{E}_{M}\left[C^{\infty}(\bar{\Omega})\right]$ satisfy the moderate slope condition.

Then there exists a solution to (3.16) in $\mathcal{E}_{M}\left[C^{\infty}(\bar{\Omega})\right]$ and thus in $\mathcal{G}\left[C^{\infty}(\bar{\Omega})\right]$.

Remark. Since there is is a canonical isomorphism $\mathcal{G}\left[C^{\infty}(\bar{\Omega})\right]$ onto $\mathcal{G}\left(C^{\infty}(\bar{\Omega})\right)$ (cf. Proposition 2 in the appendix), and every element of $\mathcal{E}_{M}\left[C^{\infty}(\bar{\Omega})\right]$ determines an element of $\mathcal{G}(\Omega)$, it follows that there exists a solution to (3.16) in $\mathcal{G}(\Omega)$.

Proof. The proof follows from Theorem 4. Fixing $k \in \mathbb{N}, k>1$, the proposed assumptions imply that there exist $\left(u_{\varepsilon}\right)_{\varepsilon} \in \mathcal{E}_{M}\left(C^{k+1, \alpha}(\bar{\Omega})\right)$ such that for a sequence $\left(\varepsilon_{k}\right)_{k} \downarrow 0$

$$
\mathcal{Q}_{\varepsilon}\left(u_{\varepsilon}^{k}\right)=0,\left.u_{\varepsilon}^{k}\right|_{\partial \Omega}=\phi_{\varepsilon},\left|u_{\varepsilon}^{k}\right|_{k+1, \alpha, \Omega}<C_{k} \varepsilon^{m_{k}}, \varepsilon<\varepsilon_{k}
$$

for suitable $C_{k}>0$ and $m_{k}<0, k \in \mathbb{N}$. Assume that $\left(C_{k}\right)_{k}$ and $\left(-m_{k}\right)_{k}$ are increasing positive sequences of numbers. Note, in (3.27) bounds for $k+1$ are larger than for $k$. But since $u_{\varepsilon}^{k+1}$ is also a fixed point for the mapping $T_{\varepsilon, \sigma}^{k}$, we have

$$
\left|u_{\varepsilon}^{k+1}\right|_{k, \alpha, \Omega}<C_{k} \varepsilon^{m_{k}}, \varepsilon<\varepsilon_{k} .
$$


Now taking

$$
u_{\varepsilon}=u_{\varepsilon}^{k}, \varepsilon \in\left[\varepsilon_{k+1}, \varepsilon_{k}\right), k \in \mathbb{N},
$$

we obtain solution $\left(u_{\varepsilon}\right)_{\varepsilon} \in \mathcal{E}_{M}\left[C^{\infty}(\bar{\Omega})\right]$. This proves the theorem.

\section{Appendix}

One can easily deduce the following version of Leray- Schauder theorem for Colombeau extensions.

Proposition 1. Let $\left(T_{\varepsilon}\right)$ be a net of mappings of $\mathcal{E}_{M}[E]$ into itself.

(i) Let $E=C^{k, \alpha}(\bar{\Omega})$, where $k$ is fixed. Assume that $T_{\varepsilon}: E \rightarrow E$ is compact, $\varepsilon<$ 1. Moreover, assume that there exist $C>0$ and $a>0$ such that the following implication holds:

$$
\begin{gathered}
\text { If } \sigma T_{\varepsilon} x_{\varepsilon}=x_{\varepsilon}, x_{\varepsilon} \in E, \varepsilon \in(0,1), \sigma \in[0,1], \\
\text { then }\left\|x_{\varepsilon}\right\| \leq C \varepsilon^{a}, \varepsilon<1 .
\end{gathered}
$$

Then $\left(T_{\varepsilon}\right)_{\varepsilon}: \mathcal{E}_{M}[E] \rightarrow \mathcal{E}_{M}[E]$ has a fixed point $\left(x_{\varepsilon}\right)_{\varepsilon} \in \mathcal{E}_{M}[E]$.

In particular, if we assume that $\left(T_{\varepsilon}\right)_{\varepsilon}$ is a net of mappings with the property

$$
T_{\varepsilon} u_{\varepsilon}-T_{\varepsilon} v_{\varepsilon} \in \mathcal{N}[E] \text { if } u_{\varepsilon}-v_{\varepsilon} \in \mathcal{N}[E],
$$

then $\left[\left(T_{\varepsilon}\right)_{\varepsilon}\right]: \mathcal{G}[E] \rightarrow \mathcal{G}[E]$ defined by

$$
\left[\left(T_{\varepsilon}\right)_{\varepsilon}\right]\left[u_{\varepsilon}\right]=\left[\left(T_{\varepsilon} u_{\varepsilon}\right)_{\varepsilon}\right],\left(u_{\varepsilon}\right)_{\varepsilon} \text { is a representative of } u \text {, }
$$

has a fixed point $x \in \mathcal{G}[E]$.

(ii) Assume that $E=C^{\infty}(\bar{\Omega})$. Put $E_{n}=C^{n, \alpha}(\bar{\Omega}), n \in \mathbb{N}$. Assume that there exists $\left(\varepsilon_{n}\right) \downarrow 0$ such that $T_{\varepsilon}: E_{n} \rightarrow E_{n} \quad$ is compact, $\varepsilon<\varepsilon_{n}, n \in \mathbf{N}$. Moreover, assume that for every $n \in \mathbb{N}$ there exist $C_{n}>0$ and $a_{n}>0$ such that the following implication holds:

$$
\begin{gathered}
\text { If } \sigma T_{\varepsilon} x_{\varepsilon}=x_{\varepsilon}, x_{\varepsilon} \in E_{n}, \varepsilon \in\left(0, \varepsilon_{n}\right), \sigma \in[0,1], \\
\text { then }\left\|x_{\varepsilon}\right\|_{n} \leq C_{n} \varepsilon^{a_{n}}, \varepsilon<\varepsilon_{n}, n \in \mathbb{N} .
\end{gathered}
$$

Then $\left(T_{\varepsilon}\right)_{\varepsilon}: \mathcal{E}_{M}[E] \rightarrow \mathcal{E}_{M}[E]$ has a fixed point $\left(x_{\varepsilon}\right)_{\varepsilon} \in \mathcal{E}_{M}[E]$.

In particular, if we assume that (4.28) holds for $\left(T_{\varepsilon}\right)_{\varepsilon}$, then we have $\left[\left(T_{\varepsilon}\right)_{\varepsilon}\right]: \mathcal{G}[E] \rightarrow \mathcal{G}[E]$ has a fixed point $x \in \mathcal{G}[E]\left(\left[\left(T_{\varepsilon}\right)_{\varepsilon}\right]\right.$ is defined in the same way as in part (i)).

Proposition 2. Let $E=C^{\infty}(\bar{\Omega})$. There is a canonical isomorphism $i$ of $\mathcal{G}[E]$ onto $\mathcal{G}(E)$.

Proof. Let $\left(R_{\varepsilon}\right)_{\varepsilon} \in \mathcal{E}_{M}[E]$. We can choose a net $\left(r_{\varepsilon}\right)_{\varepsilon} \in \mathcal{E}_{M}(E)$ and $\left(\varepsilon_{n}\right)_{n} \downarrow$ such that

$$
\left\|R_{\varepsilon}-r_{\varepsilon}\right\|_{n} \leq e^{-1 / \varepsilon}, \varepsilon \in\left(0, \varepsilon_{n}\right) .
$$

(Note, the sequence of norms $\left(\|\cdot\|_{n}\right)_{n \in \mathbb{N}}$ is increasing). Define

$$
i\left(\left(R_{\varepsilon}\right)_{\varepsilon}\right)=\left(r_{\varepsilon}\right)_{\varepsilon}, i\left(\left[\left(R_{\varepsilon}\right)_{\varepsilon}\right]\right)=\left[\left(r_{\varepsilon}\right)_{\varepsilon}\right] .
$$

This is an algebraic isomorphism. 
Remark. Consider generalized function extensions related to spaces $\left(E, \mu_{n}\right)$ and $\left(F, \nu_{n}\right)$, with increasing sequences of norms. Any continuous linear operator $h: E \rightarrow F$, can be extended to the continuous linear mapping $h: \mathcal{G}[E] \rightarrow \mathcal{G}[F]$ (with respect to the sharp topologies). Similar extensions can be made for nonlinear mappings satisfying appropriate growth conditions (cf. [4]). For example, the assumptions on $\left(T_{\varepsilon}\right)_{\varepsilon}$ which imply (4.28) can be easily formulated. Such considerations can be useful in a more general setting of nonlinear problems. In this paper we have made the concrete realization of a general concept.

\section{REFERENCES}

[1] H. A. Biagioni, A Nonlinear Theory of Generalized Functions, Springer-Verlag, BerlinHedelberg-New York, 1990.

[2] J. F. Colombeau, Elementary Introduction in New Generalized Functions, North Holland, 1985.

[3] E. De Giorgi, Sulla differenziabilità e l' analicità delle estremali degli integrali multipli regolari, Mem. Accad. Sci. Torino Cl. Sci Fis. Mat. Natur. 3: 25-43, 1957.

[4] A. Delcroix, D. Scarpalézos, Asymptotic Scales. Asymptotic Algebras, Int. Tr. Spec. Funct. 6: 181-190, 1998.

[5] C. Garetto, Pseudo-differential Operators in Algebras of Generalized Functions and Global Hypoellipticity, Acta Appl. Math. 80(2): 123-174, 2004.

[6] C. Garetto, G. Hoermann, Microlocal analysis of generalized functions: pseudodifferential techniques and propagation of singularities, Proc. Edinburgh Math. Soc. 48/3: 603-629, 2005.

[7] D. Gilbarg, N. S. Trudinger, Elliptic Partial Differential Equations of Second Order, 1 Springer-Verlag, Berlin Heidelberg New York Tokyo, 1983.

[8] M. Grosser, M. Kunzinger, M. Obrguggenberger, R. Steinbauer Geometric Generalized Functions with Applications to General Relativity, Kluwer, 2001.

[9] P. Hartmann, On the bounded slope condition, Pac. J. Math. 18: 495-511, 1966.

[10] G. Hoermann, M. de Hoop, Detection of wave front set perturbations via correlation: Foundation for wave-equation tomography, Appl. Anal. 81: 1443-1465, 2002.

[11] G. Hoermann, M. Oberguggenberger, S. Pilipović, Microlocal hypoellipticity of linear partial differential operators with generalized functions as coefficients, Trans. Amer. Math. Soc., to appear.

[12] M. Kunzinger, M. Oberguggenberger, R. Steinbauer, J. Vickers, Generalized flows and singular ODEs on differentiable manifolds, Acta Appl. Math. 80: 221-241, 2004.

[13] O. A. Ladyhenskaya, N. N. Ural'tseva, Quasilinear elliptic equations and variational problems with several independent variables, Uspehi Mat. Nauk 16: 17-92, 1961.

[14] J. Leray, J. Schauder, Topologie et equation fonctionells, Ann. Sci. Ecole Norm. Sup. 51: 17-92, 1934.

[15] D. Mitrovic, S. Pilipovic, Approximations of the linear Dirichlet problem with singular coefficients, J. Math. Anal. Appl. 313: (2006), 98-119.

[16] J. Moser, On Harnak's type theorem for elliptic differential equations, Comm. Pure Appl. Math. 14: 577-591, 1961.

[17] J. Nash, Continuity of solutions of parabolic and elliptic equations, Amer. J. Math. 80: 931-954, 1958.

[18] M. Oberguggenberger, Multiplication of Distributions and Applications to Partial Differential Equations, Pitman. Res. Not. Math. 259, Longman Sci. Techn. Essex, 1992.

[19] S. Pilipović, D. Scarpalezos, Colombeau generalized ultradistributions, Math. Proc. Cambridge Philos. Soc. 3: 541-553, 2001. 
[20] N. S. Trudinger, Local estimates for subsolutions and supersolutions of generalized of second order elliptic quasilinear equations, Invent. Math. 61: 67-79, 1980.

Department of Mathematics and Informatics, University of Novi Sad, Trg D. OBRAdovića 4, 21000 Novi SAD, SCG

E-mail address: pilipovic@im.ns.ac.yu

U.F.R. Mathematics, Case 7012, Universite Paris 7, 2 Place Jussieu, Paris, 75005 FRANCE

E-mail address: scarpalezos@math.jussieu.fr 